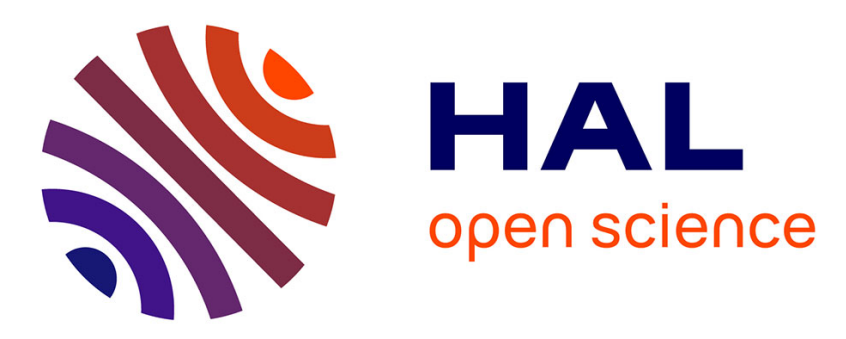

\title{
Constrained bang-bang-singular extremals
}

\author{
Laura Poggiolini, Gianna Stefani
}

\section{To cite this version:}

Laura Poggiolini, Gianna Stefani. Constrained bang-bang-singular extremals. 2019 IEEE 58th Conference on Decision and Control (CDC), Dec 2019, Nice, France. pp.1462-1467, 10.1109/CDC40024.2019.9029814 . hal-03165697

\section{HAL Id: hal-03165697 https://hal.science/hal-03165697}

Submitted on 10 Mar 2021

HAL is a multi-disciplinary open access archive for the deposit and dissemination of scientific research documents, whether they are published or not. The documents may come from teaching and research institutions in France or abroad, or from public or private research centers.
L'archive ouverte pluridisciplinaire HAL, est destinée au dépôt et à la diffusion de documents scientifiques de niveau recherche, publiés ou non, émanant des établissements d'enseignement et de recherche français ou étrangers, des laboratoires publics ou privés. 


\title{
Constrained bang-bang-singular extremals
}

\author{
Laura Poggiolini ${ }^{1,2}$ and Gianna Stefani ${ }^{1,3}$
}

\begin{abstract}
By means of Hamiltonian methods we give sufficient conditions for the strong local optimality of a Pontryagin extremal for a Mayer problem where both the end points of admissible trajectories are constrained to smooth manifolds of the state space. The extremal is given by the concatenation of two bang arcs and a partially singular one. Our sufficient conditions amount to regularity conditions on the extremal and the coercivity of a suitable quadratic form.
\end{abstract}

\section{INTRODUCTION}

The aim of this paper is to give an extension of the results obtained in [1]. In there the authors proved sufficient conditions for the strong local optimality of a Pontryagin extremal in a Mayer problem with fixed initial point and free final one and where the control set is given by a compact convex polyhedron. Here we consider the case when the Pontryagin extremal has the same control structure (i.e. bangbang-partially singular) but the constraints on the end points of admissible trajectories are completely generic. By partially singular control we mean that the control takes values in the relative interior of an edge of the polyhedron.

A precise definition of strong local optimality is given in Section I-A. Loosely speaking we can say that the localization is only with respect to the graphs of admissible trajectories, regardless of the control values. In the literature also different kinds of local optimality are considered, such as weak local optimality and Pontryagin local optimality, where the localization concerns also the control, in the $L^{\infty}$ and $L^{1}$ norms, respectively. We refer the reader to [2] for precise definitions.

In proving our result we demonstrate that the fixed-free case treated in [1] is a case study in the Hamiltonian approach. Indeed, in considering the fixed-free case, the authors prove that certain regularity assumptions on the extremal and the coercivity of a suitable second variation allow to lift any neighboring admissible trajectory to the cotangent bundle. Such lift allows to compare the cost of any such trajectory with the cost of the extremal one.

We can refer the constrained case to the free final point case thanks to the results in [3] which allow to add a penalty on the final cost. If the initial point is fixed, the lift immediately gives a comparison of the costs. Else, it

\footnotetext{
*This work was partially supported by INdAM through the GNAMPA research project 2019: "Metodi di analisi reale per l'approssimazione attraverso operatori discreti e applicazioni".

${ }^{1}$ Laura Poggiolini and Gianna Stefani are with Dipartimento di Matematica e Informatica, Università degli Studi di Firenze, via di Santa Marta 3, 50139 Firenze, Italy

2 laura.poggiolinidunifi.it

${ }^{3}$ gianna.stefani@unifi.it
}

reduces the comparison of the costs to a finite dimensional optimization problem.

We stress the fact that here we treat also the case of abnormal extremals $\left(p_{0}=0\right.$ in Pontryagin Maximum Principle) and that the problem we are considering is not a single input problem. Here we announce our result and give all the tools that are required to understand our method of proof. Detailed proofs will appear in [4]. For the sake of clarity we take the Euclidean space $\mathbb{R}^{n}$ as state space but all the results are coordinate free so that they can be easily extended to the case when the state space is a smooth manifold.

\section{A. Statement of the problem}

We consider the following optimal control problem on the fixed time interval $[0, T]$

$$
\begin{aligned}
& \text { minimize } C(\xi)=c_{0}(\xi(0))+c_{T}(\xi(T)) \text { subject to } \\
& \dot{\xi}(t) \in \mathcal{X}(\xi(t)), \quad \text { a.e. } t \in[0, T], \\
& \xi(0) \in N_{0}, \quad \xi(T) \in N_{T},
\end{aligned}
$$

where $N_{0}$ and $N_{T}$ are smooth manifolds in $\mathbb{R}^{n}$ and $\mathcal{X}(x)$ is, at each point $x \in \mathbb{R}^{n}$, the convex hull of smooth vector fields of $\mathbb{R}^{n}, X_{1}, \ldots, X_{m}$.

We assume there exists a reference trajectory $\widehat{\xi}$, whose optimality we want to investigate. More precisely the aim of the paper is to give sufficient conditions for the strong local optimality of $\widehat{\xi}$, according to the following definition:

Definition 1.1: An admissible trajectory $\widehat{\xi}:[0, T] \rightarrow \mathbb{R}^{n}$ is a strong local minimizer of problem (1) if it is a minimizer among the admissible trajectories which are close to $\widehat{\xi}$ in the $C^{0}$ topology, regardless of the control values.

We assume we know the structure of the control associated to $\widehat{\xi}$ : there exist times $\widehat{\tau}_{1}, \widehat{\tau}_{2}, 0<\widehat{\tau}_{1}<\widehat{\tau}_{2}<T$, vector fields $h_{1}, h_{2}, h_{3} \in\left\{X_{1}, \ldots X_{m}\right\}$, and a continuous function $\widehat{v} \in C^{0}\left(\left[\widehat{\tau}_{2}, T\right],(0,1)\right)$ such that $\widehat{\xi}$ is the solution to

$$
\begin{aligned}
& \dot{\xi}(t)=h_{1}(\xi(t)) \\
& \dot{\xi}(t)=h_{2}(\xi(t)) \quad t \in\left[\widehat{\tau}_{1}, \widehat{\tau}_{2}\right), \\
& \dot{\xi}(t)=\widehat{v}(t) h_{3}(\xi(t))+(1-\widehat{v}(t)) h_{2}(\xi(t)) \quad t \in\left[\widehat{\tau}_{2}, T\right], \\
& \xi(0) \in N_{0}, \quad \xi(T) \in N_{T} \text {. }
\end{aligned}
$$

Setting $f_{\mathrm{d}}:=h_{3}-h_{2}$ we can define the time-dependent reference vector field $\hat{f}_{t}$ as

$$
\widehat{f_{t}}:= \begin{cases}h_{1} & t \in\left[0, \widehat{\tau}_{1}\right), \\ h_{2} & t \in\left[\widehat{\tau}_{1}, \widehat{\tau}_{2}\right), \\ h_{2}+\widehat{v}(t) f_{\mathrm{d}} & t \in\left[\widehat{\tau}_{2}, T\right] .\end{cases}
$$

We assume that $\widehat{\xi}$ satisfies the necessary conditions for optimality, i.e. together with an adjoint covector $\widehat{\mu}:[0, T] \rightarrow$ 
$\left(\mathbb{R}^{n}\right)^{*}$, it satisfies Pontryagin Maximum Principle (PMP) and, along the singular interval $\left[\widehat{\tau}_{2}, T\right]$, it satisfies the Generalized Legendre Condition (GLC), see [5, Proposition 20.1]. Denoting $\widehat{x}_{0}:=\widehat{\xi}(0), \widehat{x}_{T}:=\widehat{\xi}(T)$, PMP and GLC can be written as follows:

Assumption 1: There exist $p_{0} \in\{0,1\}$ and an absolutely continuous mapping $\widehat{\mu}:[0, T] \rightarrow\left(\mathbb{R}^{n}\right)^{*}, p_{0}+|\widehat{\mu}(t)| \neq 0$, such that

$$
\begin{aligned}
& \dot{\widehat{\mu}}(t)=-\widehat{\mu}(t) \mathrm{D} \widehat{f}_{t}(\widehat{\xi}(t)), \\
& \left\langle\widehat{\mu}(t), \widehat{f}_{t}(\widehat{\xi}(t))\right\rangle=\max _{Y \in \mathcal{X}(\widehat{\xi}(t))}\langle\widehat{\mu}(t), Y\rangle, \quad \text { a.e. } t \in[0, T] \\
& \widehat{\mu}(0) \in p_{0} \mathrm{~d} c_{0}\left(\widehat{x}_{0}\right)+T_{\widehat{x}_{0}}^{\perp} N_{0}, \\
& \widehat{\mu}(T) \in-p_{0} \mathrm{~d} c_{T}\left(\widehat{x}_{T}\right)+T_{\widehat{x}_{T}}^{\perp} N_{T}, \\
& \left\langle\widehat{\mu}(t),\left[f_{\mathrm{d}},\left[h_{2}, f_{\mathrm{d}}\right]\right](\widehat{\xi}(t))\right\rangle \geq 0 \quad \forall t \in\left[\widehat{\tau}_{2}, T\right]
\end{aligned}
$$

where $T_{\widehat{x}_{0}}^{\perp} N_{0}$ and $T_{\widehat{x}_{T}}^{\perp} N_{T}$ are the linear spaces of oneforms which are orthogonal to the tangent spaces $T_{\widehat{x}_{0}} N_{0}$ and $T_{\widehat{x}_{T}} N_{T}$, respectively.

The trajectory $\widehat{\xi}$ of the system is called a state extremal of problem (1) while the couple $\widehat{\lambda}(t):=(\widehat{\mu}(t), \widehat{\xi}(t))$ is called an extremal of problem (1). We denote the terminal points and the switching points of the reference extremal as

$$
\widehat{\ell}_{0}:=\widehat{\lambda}(0), \quad \widehat{\ell}_{1}:=\widehat{\lambda}\left(\widehat{\tau}_{1}\right), \quad \widehat{\ell}_{2}:=\widehat{\lambda}\left(\widehat{\tau}_{2}\right), \quad \widehat{\ell}_{T}:=\widehat{\lambda}(T) .
$$

In order to prove the strong local optimality of $\widehat{\xi}$ we make further assumptions which can be split in two main categories: 1) regularity assumptions which boil down to requiring strict inequalities where the necessary conditions yield mild ones; 2) the coercivity of a second variation associated to a sub-problem of the given one.

In particular the regularity assumptions imply that $\widehat{v}$ is a smooth function so that the continuity assumption on $\widehat{v}$ is not restrictive.

\section{B. Notation}

We shall make an extensive use of notation from differential geometry which is more adequate for the Hamiltonian setting.

Given a $C^{1}$ vector field $f$ on a manifold $N$, the solution to the Cauchy problem

$$
\dot{\xi}(t)=f(\xi(t)), \quad \xi(0)=x .
$$

is denoted $\exp t f(x)$.

If $g$ is another $C^{1}$ vector field, $[f, g](x):=\mathrm{D} g(x) f(x)-$ $\mathrm{D} f(x) g(x)$ is the Lie bracket between $f$ and $g$.

For any $C^{1}$ function $a: N \rightarrow \mathbb{R}, L_{f} a(x):=$ $\langle\mathrm{d} a(x), f(x)\rangle$ is the Lie derivative of $a$ with respect to the vector field $f$ at the point $x$.

We also use some basic element of the theory of symplectic manifolds referred to the trivial cotangent bundle $T^{*} \mathbb{R}^{n} \simeq\left(\mathbb{R}^{n}\right)^{*} \times \mathbb{R}^{n}$, see for example [5].

We denote by $\pi: \ell=(p, x) \in T^{*} \mathbb{R}^{n} \mapsto x \in \mathbb{R}^{n}$ the projection on the base space. The symbol $s$ denotes the canonical Liouville one-form on $T^{*} \mathbb{R}^{n}: s:=\sum_{i=1}^{n} p^{i} \mathrm{~d} x_{i}$. The associated canonical symplectic two-form $\boldsymbol{\sigma}=\mathrm{d} \boldsymbol{s}=$ $\sum_{i=1}^{n} \mathrm{~d} p^{i} \wedge \mathrm{d} x_{i}$ allows one to associate to any, possibly time-dependent, smooth Hamiltonian $H_{t}: T^{*} \mathbb{R}^{n} \rightarrow \mathbb{R}$, a Hamiltonian vector field $\overrightarrow{H_{t}}$, by

$$
\vec{H}_{t}(\ell)=\left(-\frac{\partial H_{t}}{\partial x}(\ell), \frac{\partial H_{t}}{\partial p}(\ell)\right), \quad \forall \ell=(p, x) \in T^{*} \mathbb{R}^{n} .
$$

The maximized Hamiltonian function of the given control system is denoted as $H^{\text {max }}$ :

$$
H^{\max }(\ell):=\max _{Y \in \mathcal{X}(x)}\langle p, Y\rangle, \quad \forall \ell=(p, x) \in T^{*} \mathbb{R}^{n} .
$$

We recall that any vector field $f$ on $\mathbb{R}^{n}$ defines, by lifting to the cotangent bundle, a Hamiltonian

$$
F: \ell=(p, x) \in T^{*} \mathbb{R}^{n} \mapsto\langle p, f(x)\rangle \in \mathbb{R} .
$$

We denote by $H_{1}, H_{2}, H_{3}, F_{\mathrm{d}}$ the Hamiltonians associated with $h_{1}, h_{2}, h_{3}, f_{\mathrm{d}}$ respectively and by $H_{12}, H_{23}$ the Hamiltonians associated with the vector fields $\left[h_{1}, h_{2}\right]$ and $\left[h_{2}, h_{3}\right]$, respectively.

The solution to the Cauchy problem

$$
\frac{\mathrm{d}}{\mathrm{d} t} \xi(t)=\widehat{f}_{t}(\xi(t)), \quad \xi(T)=x,
$$

i.e. the flow from time $T$ of the reference vector field $\widehat{f}_{t}$ defined in (2) is denoted as $\widehat{S}_{t}(x)$. For any $t \in[0, T]$ the map $\widehat{S}_{t}$ is a local diffeomorphism defined in a neighborhood of the point $\widehat{x}_{T}$. Moreover

$$
\widehat{F}_{t}= \begin{cases}H_{1} & \text { if } t \in\left[0, \widehat{\tau}_{1}\right), \\ H_{2} & \text { if } t \in\left[\widehat{\tau}_{1}, \widehat{\tau}_{2}\right), \\ H_{2}+\widehat{v}(t) F_{\mathrm{d}} & \text { if } t \in\left[\widehat{\tau}_{2}, T\right],\end{cases}
$$

denotes the time-dependent reference Hamiltonian.

Finally, we identify any bilinear form $Q$ on a vector space $W$ with a linear form $Q: W \rightarrow W^{*}$, we write $Q(v, w)=\langle Q v, w\rangle$, and we denote the associate quadratic form as $Q(v, v)=Q[v]^{2}$.

\section{THE MAIN ASSUMPTIONS}

\section{A. Regularity assumptions}

For the sake of completeness we state the regularity assumptions which are the same as in [1]. We refer to that paper for more details. Along the bang arcs we require the reference Hamiltonian to be the only maximizing one (Assumption 2), while on the singular arc we require that only the Hamiltonians on the edge defined by $\mathrm{H}_{2}$ and $H_{3}$ give the maximum, and that the generalized Legendre condition holds as a strict inequality (Assumptions 3 and 4). Our last regularity assumption concerns the switching points between the different arcs of $\hat{\lambda}$ (Assumption 5).

Assumption 2 (Regularity along the bang arcs): For any $t \in\left[0, \widehat{\tau}_{1}\right)$

$$
H_{1}(\widehat{\lambda}(t))>\langle\widehat{\mu}(t), Y\rangle \quad \forall Y \in \mathcal{X}(\widehat{\xi}(t)) \backslash\left\{h_{1}(\widehat{\xi}(t))\right\},
$$

For any $t \in\left(\widehat{\tau}_{1}, \widehat{\tau}_{2}\right)$

$$
H_{2}(\widehat{\lambda}(t))>\langle\widehat{\mu}(t), Y\rangle \quad \forall Y \in \mathcal{X}(\widehat{\xi}(t)) \backslash\left\{h_{2}(\widehat{\xi}(t))\right\}
$$


Assumption 3 (Regularity along the singular arc): For any $a \in[0,1]$ and any $t \in\left[\widehat{\tau}_{2}, T\right]$

$$
\begin{aligned}
H_{2}(\widehat{\lambda}(t))+\widehat{v}(t) F_{\mathrm{d}}(\widehat{\lambda}(t))>\langle\widehat{\mu}(t), Y\rangle \\
\forall Y \in \mathcal{X}(\widehat{\xi}(t)), \quad Y \neq\left(h_{2}+a f_{\mathrm{d}}\right)(\widehat{\xi}(t)) .
\end{aligned}
$$

Assumption 4 (SGLC): For all $t \in\left[\widehat{\tau}_{2}, T\right]$

$$
R(t):=\left\langle\widehat{\mu}(t),\left[f_{\mathrm{d}},\left[h_{2}, f_{\mathrm{d}}\right]\right](\widehat{\xi}(t))\right\rangle>0 .
$$

Assumption 5 (Regularity at the switching times):

$$
\begin{gathered}
H_{12}\left(\widehat{\ell}_{1}\right)=\left\langle\widehat{\mu}\left(\widehat{\tau}_{1}\right),\left[h_{1}, h_{2}\right]\left(\widehat{\xi}\left(\widehat{\tau}_{1}\right)\right)\right\rangle>0, \\
\left\langle\widehat{\mu}\left(\widehat{\tau}_{2}\right),\left[h_{2},\left[h_{2}, f_{\mathrm{d}}\right]\right]\left(\widehat{\xi}\left(\widehat{\tau}_{2}\right)\right)\right\rangle<0 .
\end{gathered}
$$

Remark 2.1: Thanks to (3), see e.g. [1], the singular control is smooth on $\left(\widehat{\tau}_{2}, T\right)$. Moreover (3) implies that there exists a neighborhood $\mathcal{O}_{\mathrm{s}}$ of the range of the singular arc $\widehat{\lambda}\left(\left[\widehat{\tau}_{2}, T\right]\right)$ in $T^{*} \mathbb{R}^{n}$ such that the sets

$$
\begin{aligned}
\Sigma & :=\left\{\ell \in \mathcal{O}_{\mathrm{s}}: F_{\mathrm{d}}(\ell)=0\right\}, \\
\mathcal{S} & :=\left\{\ell \in \Sigma: H_{23}(\ell)=0\right\}
\end{aligned}
$$

are smooth simply connected manifolds of codimension 1 and 2 , respectively. More precisely $\overrightarrow{F_{\mathrm{d}}}$ is tangent to $\Sigma$ and transverse to $\mathcal{S}$ in $\Sigma$ and the Hamiltonian vector field $\overrightarrow{H_{23}}$ is transverse to $\Sigma$ in $\mathcal{O}_{\mathrm{s}}$, see [6].

We now recall how the regularity Assumptions 2-5 allow to define a neighborhood $\Sigma_{T}$ of $\widehat{\ell}_{T}$ in $\Sigma$ and an overmaximized Hamiltonian $H_{t}$ whose flow $\mathcal{H}$ emanating from $\Sigma_{T}$ at time $T$ satisfies the following overmaximality property

$$
H_{t} \circ \mathcal{H}_{t}(\ell) \geq H^{\max }\left(\mathcal{H}_{t}(\ell)\right) \quad(t, \ell) \in[0, T] \times \Sigma_{T},
$$

and the following compatibility conditions

$$
\begin{array}{ll}
\mathcal{H}_{t}\left(\Sigma_{T}\right) \subset \Sigma & t \in\left[\widehat{\tau}_{2}, T\right] ; \\
H_{t} \circ \widehat{\lambda}(t)=\widehat{F}_{t} \circ \widehat{\lambda}(t)=H^{\max }(\widehat{\lambda}(t)) & t \in[0, T] ; \\
\vec{H}_{t} \circ \widehat{\lambda}(t)=\vec{F}_{t} \circ \widehat{\lambda}(t) & \text { a.e. } t \in[0, T] .
\end{array}
$$

Such flow is the main tool for proving the strong local optimality of $\widehat{\xi}$. The overmaximized Hamiltonian was first introduced in [7] for singular extremals in single input control problems, then extended in [8], [9] for singular extremals in multi input control problems and in [6] for concatenations of bang and singular extremals.

Possibly restricting $\Sigma_{T}$, the following implicit function problem has a solution $\theta: \mathcal{O}_{\mathrm{s}} \rightarrow \mathbb{R}$ :

$$
\left\{\begin{array}{l}
H_{23} \circ \exp \left(\theta(\ell) \overrightarrow{F_{\mathrm{d}}}\right)(\ell)=0 \\
\theta(\ell)=0 \quad \text { if } H_{23}(\ell)=0
\end{array}\right.
$$

Starting from

$$
\widetilde{H}_{2}(\ell):=H_{2} \circ \exp \left(\theta(\ell) \overrightarrow{F_{\mathrm{d}}}\right)(\ell)
$$

we define, for $t \in\left[\widehat{\tau}_{2}, T\right]$ the over-maximized Hamiltonian as

$$
H_{t}(\ell):=\widetilde{H}_{2}(\ell)+\widehat{v}(t) F_{\mathrm{d}}(\ell)
$$

The main properties of $\widetilde{H}_{2}$ and $H_{t}$ are summarized in the following lemma, proven in [6].
Lemma 2.1: Possibly restricting $\mathcal{O}_{\mathrm{s}}$ the following properties hold

1) $\widetilde{H}_{2}(\ell) \geq H_{2}(\ell)$ for any $\ell \in \Sigma$ and equality holds if and only if $\ell \in \mathcal{S}$.

2) $\overrightarrow{\widetilde{H}_{2}}$ and $\overrightarrow{H_{t}}$ are tangent to $\Sigma$.

To extend $H_{t}$ to the bang intervals we need to define the switching times near the reference switching points $\widehat{\ell}_{1}$ and $\widehat{\ell}_{2}$. Thanks to the second inequality in Assumption 5, the implicit function theorem applies to the problem

$$
\left\{\begin{array}{l}
H_{23} \circ \exp \left(t_{2}-\widehat{\tau}_{2}\right) \overrightarrow{\widetilde{H}}_{2}(\ell)=0, \\
t_{2}(\ell)=\widehat{\tau}_{2} \quad \text { if } H_{23}(\ell)=0,
\end{array}\right.
$$

so that it defines a real valued function $t_{2}$ in a neighborhood $\mathcal{O}_{2}$ of $\widehat{\ell}_{2}$. If $\ell \in \Sigma$, then $t_{2}(\ell)=\widehat{\tau}_{2}$ if and only if $\ell \in \mathcal{S}$.

We set

$$
\tau_{2}(\ell):=\min \left\{t_{2}(\ell), \widehat{\tau}_{2}\right\}= \begin{cases}t_{2}(\ell) & \text { if } H_{23}(\ell)<0, \\ \widehat{\tau}_{2} & \text { if } H_{23}(\ell) \geq 0 .\end{cases}
$$

Finally, possibly shrinking $\mathcal{O}_{2}$, we can define the switching time $\tau_{1}: \mathcal{O}_{2} \rightarrow \mathbb{R}$. Indeed, thanks to the first inequality in Assumption 5, the implicit function theorem applies to

$$
\left\{\begin{array}{l}
\left(H_{2}-H_{1}\right) \circ \exp \left(\tau_{1}-\tau_{2}(\ell)\right) \overrightarrow{H_{2}} \circ \\
\quad \circ \exp \left(\tau_{2}(\ell)-\widehat{\tau}_{2}\right){\overrightarrow{H_{2}}}_{2}(\ell)=0, \\
\tau_{1}\left(\widehat{\ell}_{2}\right)=\widehat{\tau}_{1},
\end{array}\right.
$$

see e.g. [10].

Possibly restricting $\Sigma_{T}$ we can now extend the flow $(t, \ell) \mapsto \mathcal{H}_{t}(\ell)$ emanating from $\Sigma_{T}$ at time $T$ backwards in time.

For $t<\widehat{\tau}_{2}$, setting $\tilde{\ell}:=\mathcal{H}_{\widehat{\tau}_{2}}(\ell)$, we define

$\mathcal{H}_{t}(\ell):= \begin{cases}\exp \left(t-\widehat{\tau}_{2}\right) \overrightarrow{\widetilde{H}_{2}}(\widetilde{\ell}) & t \in\left[\tau_{2}(\widetilde{\ell}), \widehat{\tau}_{2}\right], \\ \exp \left(t-\tau_{2}(\widetilde{\ell})\right) \overrightarrow{H_{2}} \circ \mathcal{H}_{\tau_{2}(\widetilde{\ell})}(\widetilde{\ell}) & t \in\left[\tau_{1}(\widetilde{\ell}), \tau_{2}(\widetilde{\ell})\right), \\ \exp \left(t-\tau_{1}(\widetilde{\ell})\right) \overrightarrow{H_{1}} \circ \mathcal{H}_{\tau_{1}(\widetilde{\ell})}(\widetilde{\ell}) & t \in\left[0, \tau_{1}(\widetilde{\ell})\right) .\end{cases}$

\section{B. The extended second variation}

In order to use the Hamiltonian approach we first extend in a suitable way the cost functions $p_{0} c_{0}$ and $p_{0} c_{T}$ defined on $N_{0}$ and $N_{T}$, respectively. Indeed they can be extended to the whole $\mathbb{R}^{n}$ in such a way that the transversality conditions hold on the whole tangent spaces. Let $\alpha, \beta: \mathbb{R}^{n} \rightarrow \mathbb{R}$ be smooth functions such that

$$
\begin{array}{ll}
\beta=p_{0} c_{T} \text { on } N_{T}, & \widehat{\mu}(T)=\mathrm{d}(-\beta)\left(\widehat{x}_{T}\right), \\
\alpha=p_{0} c_{0} \text { on } N_{0}, & \widehat{\mu}(0)=\mathrm{d} \alpha\left(\widehat{x}_{0}\right) .
\end{array}
$$

In the normal case $\left(p_{0}=1\right) \beta$ and $\alpha$ are cost functions equivalent to the original ones while in the abnormal case $\left(p_{0}=0\right)$ they are extensions of the zero function. When $p_{0}=$ 0 all the costs disappear and we are studying the constraints. Proving that $\widehat{\xi}$ is a strict strong minimizer with $p_{0}=0$ means that it is an isolated admissible trajectory.

We consider the problem of minimizing the cost

$$
\alpha(\xi(0))+\beta(\xi(T)),
$$


allowing only for perturbations of the reference control on the singular interval $\left(\widehat{\tau}_{2}, T\right)$ and for perturbations of $\widehat{\tau}_{1}$.

In order to write the second variation, as in [1], we pushforward the problem to the time $T$. Indeed we define

$$
\begin{array}{ll}
\widehat{\alpha}:=\alpha \circ \widehat{S}_{0}, & \widehat{N}_{0}:=\widehat{S}_{0}^{-1}\left(N_{0}\right), \\
g_{t}:=\widehat{S}_{t *}^{-1} f_{\mathrm{d}} \circ \widehat{S}_{t}, & t \in\left[\widehat{\tau}_{2}, T\right], \\
k_{i}:=\widehat{S}_{\widehat{\tau}_{1} *}^{-1} h_{i} \circ \widehat{S}_{\widehat{\tau}_{1}}, & i=1,2, \quad k:=k_{1}-k_{2} .
\end{array}
$$

We thus obtain a problem evolving in a neighborhood of $\widehat{x}_{T}$

$$
\begin{aligned}
& \text { minimize } \widehat{\alpha}(\eta(0))+\beta(\eta(T)) \text { subject to } \\
& \dot{\eta}(t)= \begin{cases}\left(v_{0}(t)-1\right) k_{1}(\xi(t)) & t \in\left(0, \widehat{\tau}_{1}\right), \\
\left(v_{0}(t)-1\right) k_{2}(\xi(t)) & t \in\left(\widehat{\tau}_{1}, \widehat{\tau}_{2}\right), \\
(v(t)-\widehat{v}(t)) g_{t}(\eta(t)) & t \in\left(\widehat{\tau}_{2}, T\right),\end{cases} \\
& v_{0}(t)>0, \int_{0}^{\widehat{\tau}_{2}}\left(v_{0}(t)-1\right) \mathrm{d} t=0, \\
& |v(t)-\widehat{v}(t)|<\delta, \quad \eta(0) \in \widehat{N}_{0}, \quad \eta(T) \in N_{T},
\end{aligned}
$$

where $\delta$ is a suitable positive constant.

As in [1] we first write the second variation of problem (6) and then, by means of an intrinsic version of a Goh transformation, we extend it to a new quadratic form which we call extended second variation.

Denoting $\delta e:=\left(\delta x, \delta y, \varepsilon_{0}, \varepsilon_{1}, w\right) \in T_{\widehat{x}_{T}} N_{T} \times T_{\widehat{x}_{T}} \widehat{N}_{0} \times$ $\mathbb{R} \times \mathbb{R} \times L^{2}\left(\left[\widehat{\tau}_{2}, T\right]\right)$, the extended second variation of (6) is the linear quadratic form defined by

$$
\begin{aligned}
& J_{\text {ext }}[\delta e]^{2}=\frac{1}{2} \mathrm{D}^{2}(\widehat{\alpha}+\beta)\left(\widehat{x}_{T}\right)[\delta y]^{2} \\
& +\frac{\varepsilon_{0}^{2}}{2}\left(L_{k}^{2} \beta\left(\widehat{x}_{T}\right)+L_{\left[k_{2}, k_{1}\right]} \beta\left(\widehat{x}_{T}\right)\right) \\
& +\varepsilon_{0} L_{\delta y} L_{k} \beta\left(\widehat{x}_{T}\right)-\frac{\varepsilon_{1}^{2}}{2} L_{f_{\mathrm{d}}}^{2} \beta\left(\widehat{x}_{T}\right)-\varepsilon_{1} L_{\delta x} L_{f_{\mathrm{d}}} \beta\left(\widehat{x}_{T}\right) \\
& +\frac{1}{2} \int_{\widehat{\tau}_{2}}^{T}\left(2 w(t) L_{\zeta(t)} L_{\dot{g}_{t}} \beta\left(\widehat{x}_{T}\right)+w(t)^{2} R(t)\right) \mathrm{d} t
\end{aligned}
$$

subject to

$$
\begin{gathered}
\dot{\zeta}(t)=w(t) \dot{g}_{t}\left(\widehat{x}_{T}\right), \quad t \in\left[\widehat{\tau}_{2}, T\right] \\
\zeta\left(\widehat{\tau}_{2}\right)=\delta y+\varepsilon_{0} k\left(\widehat{x}_{T}\right), \quad \zeta(T)=\delta x+\varepsilon_{1} f_{\mathrm{d}}\left(\widehat{x}_{T}\right) .
\end{gathered}
$$

We denote by $\mathcal{W}_{\text {ext }}$ the space of admissible variations of the above described problem.

Remark 2.2: Notice that $\dot{g}_{t}=\widehat{S}_{t *}^{-1}\left[h_{2}, h_{3}\right] \circ \widehat{S}_{t}, \quad t \in$ $\left[\widehat{\tau}_{2}, T\right]$ and $L_{\left[k_{2}, k_{1}\right]} \beta\left(\widehat{x}_{T}\right)=H_{12}\left(\widehat{\ell}_{1}\right)$. Moreover $J_{\text {ext }}$ does not depend on the choice of the functions $\alpha$ and $\beta$ satisfying (4)-(5).

\section{THE MAIN RESULTS}

We can now state the main theorems of this paper.

Theorem 3.1: Assume that the reference trajectory $\widehat{\xi}$ is a state extremal (Assumption 1) satisfying the regularity Assumptions 2-5. Assume the extended second variation $J_{\text {ext }}$ is coercive.

- If $p_{0}=1$, then $\widehat{\xi}$ is a strict strong local optimal trajectory of (1).
- If $p_{0}=0$, then $\mathcal{W}_{\text {ext }}=\{0\}$ and $\widehat{\xi}$ is an isolated admissible trajectory of (1a)-(1b).

We can also prove strong local optimality in a case when the extended second variation is not coercive.

Theorem 3.2: Assume that the reference trajectory $\widehat{\xi}$ is a state extremal (Assumption 1) satisfying the regularity Assumptions 2-5. Assume

i) $f_{\mathrm{d}}\left(\widehat{x}_{T}\right) \neq 0$;

ii) There exists a neighborhood $\mathcal{O}$ of $\widehat{x}_{T}$ such that

$$
f_{\mathrm{d}}(x) \in T_{x} N_{T}, L_{f_{\mathrm{d}}}\left(p_{0} c_{T}\right)(x)=0 \quad \forall x \in \mathcal{O} \cap N_{T} .
$$

iii) The extended second variation $J_{\text {ext }}$ restricted to $\left\{\delta e \in \mathcal{W}_{\text {ext }}: \varepsilon_{1}=0\right\}$ is coercive.

Then

- in the normal case $p_{0}=1, \widehat{\xi}$ is a strict strong local optimal trajectory of (1);

- in the abnormal case $p_{0}=0, \widehat{\xi}$ is an isolated admissible trajectory of (1a)-(1b).

Indeed in both the main results of this paper, we deal with the coercivity of a quadratic form of the following kind

$$
\begin{aligned}
& J_{\text {ext }}\left[\left(\delta x, \delta y, \varepsilon_{0}, w\right)\right]^{2}=\frac{1}{2} \mathrm{D}^{2}(\widehat{\alpha}+\widetilde{\beta})\left(\widehat{x}_{T}\right)[\delta y]^{2} \\
& +\frac{\varepsilon_{0}^{2}}{2}\left(L_{k}^{2} \widetilde{\beta}\left(\widehat{x}_{T}\right)+H_{12}\left(\widehat{\ell}_{1}\right)\right)+\varepsilon_{0} L_{\delta y} L_{k} \widetilde{\beta}\left(\widehat{x}_{T}\right) \\
& +\frac{1}{2} \int_{\widehat{\tau}_{2}}^{T}\left(2 w(t) L_{\zeta(t)} L_{\dot{g}_{t}} \widetilde{\beta}\left(\widehat{x}_{T}\right)+w(t)^{2} R(t)\right) \mathrm{d} t
\end{aligned}
$$

subject to

$$
\begin{gathered}
\dot{\zeta}(t)=w(t) \dot{g}_{t}\left(\widehat{x}_{T}\right), \\
\zeta\left(\widehat{\tau}_{2}\right)=\delta y+\varepsilon_{0} k\left(\widehat{x}_{T}\right), \quad \zeta(T)=\delta x \in V_{T}
\end{gathered}
$$

where $V_{T}$ is a suitable linear subspace of $\mathbb{R}^{n}$ and $\widetilde{\beta}$ is a smooth function defined in a neighborhood $\mathcal{O}$ of $\widehat{x}_{T}$, satisfying the following properties

$$
\begin{aligned}
& \widetilde{\beta}(x) \leq \beta(x) \forall x \in \mathcal{O}, \quad \widetilde{\beta}\left(\widehat{x}_{T}\right)=\beta\left(\widehat{x}_{T}\right), \\
& L_{f_{\mathrm{d}}} \widetilde{\beta}(x)=0 \forall x \in \mathcal{O}, \quad \mathrm{d} \widetilde{\beta}\left(\widehat{x}_{T}\right)=-\widehat{\mu}(T) .
\end{aligned}
$$

$V_{T}$ and $\widetilde{\beta}$ are defined according to the different cases examined in the discussion below. Theorem 3.1 is covered by cases 1) and 2) while case 3 ) is the one considered in Theorem 3.2.

1) $f_{\mathrm{d}}\left(\widehat{x}_{T}\right) \in T_{\widehat{x}_{T}} N_{T}$. Evaluating the second variation on $\delta e=\left(-f_{\mathrm{d}}\left(\widehat{x}_{T}\right), 0,0,1,0\right)$ we obtain $J_{\text {ext }}[\delta e]^{2}=$ $\frac{1}{2} L_{f_{\mathrm{d}}}^{2} \beta\left(\widehat{x}_{T}\right)$ so that a necessary condition for the coercivity of $J_{\text {ext }}$ is $L_{f_{\mathrm{d}}}^{2} \beta\left(\widehat{x}_{T}\right)>0$. This condition does not depend on the choice of $\beta$ satisfying the compatibility conditions (4) and it implies that we are dealing with the normal case $p_{0}=1$.

In this case we consider the set, locally defined near $\widehat{x}_{T}$ in $\mathbb{R}^{n}$,

$$
\widetilde{M}:=\left\{x \in \mathbb{R}^{n}: L_{f_{\mathrm{d}}} \beta(x)=0\right\} .
$$

$\widetilde{M}$ is a hyper-surface whose tangent space at $\widehat{x}_{T}$ is

$$
T_{\widehat{x}_{T}} \widetilde{M}=\left\{\delta z \in \mathbb{R}^{n}: L_{\delta z} L_{f_{\mathrm{d}}} \beta\left(\widehat{x}_{T}\right)=0\right\}
$$


and the intersection $\widetilde{N}_{T}:=\widetilde{M} \cap N_{T}$ is a submanifold of $\mathbb{R}^{n}$ whose tangent space at $\widehat{x}_{T}$ is

$$
T_{\widehat{x}_{T}} \widetilde{N}_{T}=\left\{\delta z \in N_{T}: L_{\delta z} L_{f_{\mathrm{d}}} \beta\left(\widehat{x}_{T}\right)=0\right\} .
$$

Notice that $\widetilde{M}$ depends on the choice of $\beta$ but $\widetilde{N}_{T}$ does not. For $x=\exp \left(r f_{\mathrm{d}}\right)(z), z \in \widetilde{M}$ set $\widetilde{\beta}(x):=\beta(z)$, i.e. we extend $\left.\beta\right|_{\widetilde{M}}$ as a constant function along the integral lines of $f_{\mathrm{d}}$.

Setting $V_{T}:=T_{\widehat{x}_{T}} N_{T}$ we obtain that the coercivity of $J_{\text {ext }}$ is equivalent to $L_{f_{\mathrm{d}}}^{2} \beta\left(\widehat{x}_{T}\right)>0$ plus the coercivity of the quadratic form (7)-(8), see [1] for details.

2) $f_{\mathrm{d}}\left(\widehat{x}_{T}\right) \notin T_{\widehat{x}_{T}} N_{T}$. In this case we can choose $\beta$ with properties (4) and such that $L_{f_{\mathrm{d}}} \beta \equiv 0$. Since $\beta$ satisfies the properties of $\widetilde{\beta}$ in (9), we set $\widetilde{\beta}:=\beta$, $V_{T}:=T_{\widehat{x}_{T}} N_{T} \oplus \mathbb{R} f_{\mathrm{d}}\left(\widehat{x}_{T}\right)$ and we obtain for $J_{\text {ext }}$ the same formulas as in (7)-(8).

3) If assumptions i) and ii) of Theorem 3.2 are satisfied, then $J_{\text {ext }}\left[\left(f_{\mathrm{d}}\left(\widehat{x}_{T}\right), 0,0,-1,0\right)\right]^{2}=0$, so that $J_{\text {ext }}$ is not coercive. By the properties of $f_{\mathrm{d}}$, we can choose the extension $\beta$ such that $L_{f_{\mathrm{d}}} \beta \equiv 0$ and require the coercivity on the subspace of $\mathcal{W}_{\text {ext }}$ such that $\varepsilon_{1}=0$. In this case $\beta$ satisfies the properties of $\widetilde{\beta}$ in (9) so that, if we set $\widetilde{\beta}:=\beta$ and $V_{T}:=T_{\widehat{x}_{T}} N_{T}$, we obtain for $J_{\text {ext }}$ the same formulas as in (7)-(8).

\section{PROOF OF THE RESUlT}

\section{A. A Hamiltonian sufficient condition}

We now describe the Hamiltonian methods that we are going to use to prove our strong local optimality result. Assume $\widetilde{c}$ is a smooth function such that

$$
\begin{array}{ll}
\widetilde{c}\left(\widehat{x}_{T}\right)=\widetilde{\beta}\left(\widehat{x}_{T}\right), & \mathrm{d} \widetilde{c}\left(\widehat{x}_{T}\right)=\mathrm{d} \widetilde{\beta}\left(\widehat{x}_{T}\right), \\
\widetilde{c} \geq \widetilde{\beta}, & L_{f_{\mathrm{d}}} \widetilde{c} \equiv 0 .
\end{array}
$$

Define the Lagrangian manifold

$$
\Lambda:=\{(\mathrm{d}(-\widetilde{c})(x), x): x \in \mathcal{O}\}
$$

contained in $\Sigma$ and let $T_{\widehat{\ell}_{T}} \Lambda$ be its tangent space at $\widehat{\ell}_{T}$. Consider the problem

$$
\begin{aligned}
& \operatorname{minimize} \widetilde{C}(\xi):=\alpha(\xi(0))+\widetilde{c}(\xi(T)) \text { subject to } \\
& \dot{\xi}(t) \in \mathcal{X}(\xi(t)) \quad \text { a.e. } t \in[0, T], \\
& \xi(0) \in N_{0}, \quad \xi(T) \in \mathbb{R}^{n} .
\end{aligned}
$$

Our main tool for proving the strong local optimality result is the following theorem, whose proof is given in [4].

Theorem 4.1: Let $\Lambda$ be defined in (11) and let $H_{t}$ be the overmaximized Hamiltonian defined in Section II-A. Define $\mathrm{d} \alpha_{*}:=\mathrm{D}^{2} \alpha\left(\widehat{x}_{0}\right), \mathcal{H}_{0 *}:=\mathrm{D} \mathcal{H}_{0}\left(\widehat{\ell}_{T}\right)$ and assume

1) The map

$$
\text { id } \times \pi \mathcal{H}:(t, \ell) \in[0, T] \times \Lambda \mapsto\left(t, \pi \mathcal{H}_{t}(\ell)\right) \in \mathcal{U}
$$

is locally Lipschitz invertible onto a neighborhood $\mathcal{U}$ of the graph of $\widehat{\xi}$ in $[0, T] \times \mathbb{R}^{n}$,

2) $\boldsymbol{\sigma}\left(\mathrm{d} \alpha_{*} \pi \mathcal{H}_{0 *} \delta \ell, \mathcal{H}_{0 *} \delta \ell\right)>0$ for any $\delta \ell \in T_{\widehat{\ell}_{T}} \Lambda$ such that $\pi \mathcal{H}_{0 *} \delta \ell \in T_{\widehat{x}_{0}} N_{0}$.

Then $\widehat{\xi}$ is a strict strong locally optimal trajectory for the cost $\widetilde{C}(\xi)$ subject to (12a)-(12b).

\section{B. Reduction to a free final point problem}

We are now going to define the function $\widetilde{c}$. The main tool is [3, Theorem 13.2] which permits to extend coercive quadratic forms defined on a closed linear subspace of a Hilbert space preserving the coercivity.

Let $p$ be the dimension of the linear space $V_{T}$ and let $f_{2}, \ldots f_{p}: \mathbb{R}^{n} \rightarrow T \mathbb{R}^{n}$ be smooth vector fields such that

$$
\operatorname{Span}\left\{f_{\mathrm{d}}\left(\widehat{x}_{T}\right), f_{2}\left(\widehat{x}_{T}\right), \ldots f_{p}\left(\widehat{x}_{T}\right)\right\}=V_{T}
$$

and choose coordinates in $\mathbb{R}^{n}$ by

$$
x \mapsto \exp x^{1} f_{\mathrm{d}} \circ x^{2} f_{2} \ldots \circ \exp x^{p} f_{p} \circ \ldots \circ \exp x^{n} f_{n}\left(\widehat{x}_{T}\right) .
$$

Let $\psi: \mathbb{R}^{n} \rightarrow \mathbb{R}$ be defined as $\psi(x):=\frac{1}{2} \sum_{i=p+1}^{n}\left(x^{i}\right)^{2}$ and consider the system

$$
\begin{gathered}
\dot{\zeta}(t)=w(t) \dot{g}_{t}\left(\widehat{x}_{T}\right), \\
\zeta\left(\widehat{\tau}_{2}\right)=\delta y+\varepsilon_{0} k\left(\widehat{x}_{T}\right), \quad \zeta(T)=\delta x \in \mathbb{R}^{n} .
\end{gathered}
$$

In the Hilbert space

$$
\mathcal{V}:=\left\{\left(\delta x, \delta y, \varepsilon_{0}, w\right) \in \mathbb{R}^{n} \times T_{\widehat{x}_{T}} \widehat{N}_{0} \times \mathbb{R} \times L^{2}\left(\left[\widehat{\tau}_{2}, T\right]\right):\right.
$$

(13) admits a solution $\}$

we apply [3, Theorem 13.2] to the Legendre form $J_{\text {ext }}$ given by (7) and to the weakly continuous quadratic form $\mathrm{D}^{2} \psi\left(\widehat{x}_{T}\right)[\delta e]^{2}:=\mathrm{D}^{2} \psi\left(\widehat{x}_{T}\right)[\delta x]^{2}$. There exists $\rho>0$ such that

$$
J\left[\delta x, \delta y, \varepsilon_{0}, w\right]^{2}:=J_{\text {ext }}\left[\delta x, \delta y, \varepsilon_{0}, w\right]^{2}+\rho \mathrm{D}^{2} \psi\left(\widehat{x}_{T}\right)[\delta x]^{2}
$$

is coercive on $\mathcal{V}$. Let

$$
\begin{gathered}
\widetilde{c}:=\widetilde{\beta}+\rho \psi, \quad J_{0}:=L_{k}^{2} \widetilde{c}\left(\widehat{x}_{T}\right)+H_{12}\left(\widehat{\ell}_{1}\right), \\
\gamma^{\prime \prime}:=\mathrm{D}^{2}(\widehat{\alpha}+\widetilde{c})\left(\widehat{x}_{T}\right) .
\end{gathered}
$$

Since, by (13), $\delta x=\delta y+\varepsilon_{0} k\left(\widehat{x}_{T}\right)+\int_{\widehat{\tau}_{2}}^{T} w(t) \dot{g}_{t}\left(\widehat{x}_{T}\right) \mathrm{d} t$, with lengthy calculations, which will be given in [4], we obtain

$$
\begin{aligned}
& 2 J\left[\delta x, \delta y, \varepsilon_{0}, w\right]^{2}=\gamma^{\prime \prime}[\delta y]^{2}+2 \varepsilon_{0} L_{\delta y} L_{k} \widetilde{c}\left(\widehat{x}_{T}\right)+ \\
& +\varepsilon_{0}^{2} J_{0}+\int_{\widehat{\tau}_{2}}^{T}\left(2 w(t) L_{\zeta(t)} L_{\dot{g}_{t}} \widetilde{c}\left(\widehat{x}_{T}\right)+w(t)^{2} R(t)\right) \mathrm{d} t
\end{aligned}
$$

subject to (13).

Remark 4.2: The function $\widetilde{c}$ defined above satisfies the properties required in (10) and $\widehat{\lambda}$ is a normal Pontryagin extremal for the optimal control problem (12).

The coercivity of $J$ on $\mathcal{V}$ is equivalent to requiring the coercivity of $J$ on the space

$$
\mathcal{V}_{0}:=\left\{\delta e=\left(\delta x, \delta y, \varepsilon_{0}, w\right) \in \mathcal{V}: \delta y=0\right\}
$$

and the coercivity of $J$ on $\mathcal{V}_{0}^{\perp J}$, i.e. on the orthogonal with respect to $J$ of $\mathcal{V}_{0}$ in $\mathcal{V}$.

In [1] the authors prove that the coercivity of $J$ on $\mathcal{V}_{0}$ implies that the first assumption of Theorem 4.1 is satisfied.

In order to prove that also the second assumption is satisfied, one has to exploit the coercivity of $J$ on $\mathcal{V}_{0}^{\perp_{J}}$. Here we only sketch the proof and we refer the reader to [4] for details. Indeed, let $\left(\mu_{t}^{\prime \prime}(\delta x), \zeta_{t}^{\prime \prime}(\delta x)\right)$ be the solution to 
the Hamiltonian linear system associated with the quadratic Hamiltonian

$$
H_{t}^{\prime \prime}(\delta p, \delta x)=-\frac{1}{2 R(t)}\left(\left\langle\delta p, \dot{g}_{t}\left(\widehat{x}_{T}\right)\right\rangle+L_{\delta x} L_{\dot{g}_{t}} \widetilde{c}\left(\widehat{x}_{T}\right)\right)^{2}
$$

and final conditions $\left(\mu_{T}^{\prime \prime}(\delta x), \zeta_{T}^{\prime \prime}(\delta x)\right)=(0, \delta x)$. Writing the bilinear form associated with $J$, one can prove that if $\delta e=\left(\delta x, \delta y, \varepsilon_{0}, w\right)$ is in $V_{0}^{\perp_{J}}$ then

$$
\begin{gathered}
\varepsilon_{0} H_{12}\left(\widehat{\ell}_{1}\right)+L_{\zeta_{\widehat{\tau}_{2}}^{\prime \prime}(\delta x)} L_{k} \widetilde{c}\left(\widehat{x}_{T}\right)+\left\langle\mu_{\widehat{\tau}_{2}}^{\prime \prime}(\delta x), k\left(\widehat{x}_{T}\right)\right\rangle=0 \\
2 J[\delta e]^{2}=\gamma^{\prime \prime}[\delta y]^{2}+\varepsilon_{0} L_{\delta y} L_{k} \widetilde{c}\left(\widehat{x}_{T}\right)+\left\langle\mu_{\widehat{\tau}_{2}}^{\prime \prime}(\delta x), \delta y\right\rangle .
\end{gathered}
$$

By means of the antisymplectic isomorphism

$$
\begin{gathered}
\iota:\left(\mathbb{R}^{n}\right)^{*} \times \mathbb{R}^{n} \rightarrow\left(\mathbb{R}^{n}\right)^{*} \times \mathbb{R}^{n}, \\
\iota:(\delta p, \delta x) \mapsto \delta \ell:=\left(-\delta p+\mathrm{D}^{2}(-\widetilde{\beta})\left(\widehat{x}_{T}\right)(\delta x, \cdot), \delta x\right)
\end{gathered}
$$

one can show that for any $\delta \ell \in T_{\widehat{\ell}_{T}} \Lambda$, there holds

$$
\boldsymbol{\sigma}\left(\mathrm{d} \alpha_{*} \pi \mathcal{H}_{0 *} \delta \ell, \mathcal{H}_{0 *} \delta \ell\right)=2 J[\delta e]^{2}
$$

where $\delta e=\left(\delta x, \delta y, \varepsilon_{0}, w\right) \in V_{0}^{\perp_{J}}$ is defined by

$$
\delta x=\pi_{*} \delta \ell, \delta y=\widehat{S}_{0 *}^{-1}\left(\pi \mathcal{H}_{0}\right)_{*} \delta \ell, \varepsilon_{0}=\left\langle\mathrm{d} \tau_{1}\left(\widehat{\ell}_{2}\right), \mathcal{H}_{\widehat{\tau}_{2 *}} \delta \ell\right\rangle
$$

and $w$ is the extremal control associated to the LQ accessory problem (13)-(14).

Summarizing, assuming the coercivity of (7)-(8), we obtain the coercivity of (13)-(14) so that also assumption 2 of Theorem 4.1 holds. Thus Theorems 3.1-3.2 are proved.

\section{CONCLusion}

We point out that the case we have studied corresponds to a multi-input controlled dynamics where the control set is the unit symplex of $\mathbb{R}^{m}$ and where the extremal control takes values either in a vertex of the control set or in the relative interior of one of its one-dimensional edges.

It is clear to the authors that the result can be easily extended to the case of bang-bang-singular (or singular-bangbang) concatenations with an arbitrary number of bang arcs.

Our approach was applied successfully to the minimum time problem in the case of bang-singular-bang extremals, see [6], and bang-singular ones, see [11], thus covering also the Dubin's problem and the dodgem car problem.

The Mayer problem for bang-singular-bang concatenations presents some technical difficulties and it is still under study.

The present paper extends the results proved by the authors in [1]. In there the authors also studied a Van Der Pol oscillator and proved that the assumptions of Theorem 3.2 are satisfied by the extremal found in [12] (also studied in [13]). This example is particularly meaningful as the strong local optimality of the extremal had not yet been proved. Indeed in [13] the author proved that the extremal is optimal among trajectories having the same bang-bang-singular structure with different switching times. We point out that the example fits in the class where the extended second variation is not coercive.

Other examples, fitting in the same non-coercive class, are provided in [14, Section IV.C and IV.D] for an appropriate cost function and appropriate choices of the control set with the dynamic constraint given by the Rayleigh equation.

The authors are currently studying structural stability of bang-bang-singular extremals, by using the Hamiltonian approach as in [15] for the minimum time problem.

\section{REFERENCES}

[1] L. Poggiolini and G. Stefani, "Strong local optimality for a bang-bang-singular extremal: the fixed-free case," SIAM J. Control Optim., vol. 56, no. 3, pp. 2274-2294, 2018. [Online]. Available: https://doi.org/10.1137/17M1140248

[2] A. A. Milyutin and N. P. Osmolovskii, Calculus of Variations and Optimal Control, ser. Translations of Mathematical Monographs. American Mathematical Society, 1998, vol. 180.

[3] M. R. Hestenes, "Applications of the theory of quadratic forms in Hilbert space to calculus of variations," Pacific J. Math, vol. 1, pp. 525-581, 1951.

[4] L. Poggiolini and G. Stefani, "Strong local optimality for a bang-bangsingular extremal: general constraints," work in progress.

[5] A. A. Agrachev and Y. L. Sachkov, Control Theory from the Geometric Viewpoint. Springer-Verlag, 2004.

[6] L. Poggiolini and G. Stefani, "Bang-singular-bang extremals: sufficient optimality conditions," Journal of Dynamical and Control Systems, vol. 17, pp. 469-514, 2011. [Online]. Available: http://dx.doi.org/10.1007/s10883-011-9127-y

[7] G. Stefani, "Strong optimality of singular trajectories," in Geometric Control and Nonsmooth Analysis, ser. Series on Advances in Mathematics for Applied Sciences, F. Ancona, A. Bressan, P. Cannarsa, F. Clarke, and P. R. Wolenski, Eds., vol. 76. Hackensack, NJ: World Scientific Publishing Co. Pte. Ltd., 2008, pp. 300-326.

[8] F. C. Chittaro and G. Stefani, "Singular extremals in multi-input timeoptimal problem: a sufficient condition," Control and Cybernetics, vol. 39 , no. 4 , p. to appear, 2010.

[9] _ - "Minimum-time strong optimality of a singular arc: The multiinput non involutive case," ESAIM: COCV, vol. 22, no. 3, pp. 786-810, 2016. [Online]. Available: http://dx.doi.org/10.1051/cocv/2015026

[10] A. A. Agrachev, G. Stefani, and P. Zezza, "Strong optimality for a bang-bang trajectory," SIAM J. Control Optimization, vol. 41, no. 4 , pp. 991-1014, 2002.

[11] L. Poggiolini and G. Stefani, "A case study in strong optimality and structural stability of bang-singular extremals," in Geometric Control Theory and sub-Riemannian Geometry, G. Stefani, U. Boscain, J.-P. Gauthier, and A. Sarychev, Eds. Springer-Verlag, 2013, pp. 333-350. [Online]. Available: http://www.springer.com/mathematics/book/9783-319-02131-7

[12] H. Maurer, "Theory and applications of bang-bang and singular control problems," Conference slides, 2007, 23rd IFIP TC 7 Conference on System Modelling and Optimization Kraków, Poland, July 23-27, 2007.

[13] G. Vossen, "Switching time optimization for bang-bang and singular controls," Journal of Optimization Theory and Applications, vol. 144, no. 2, pp. 409-429, 2010.

[14] H. Maurer and N. Osmolovskii, "Second-order conditions for optimal control problems with mixed control-state constraints and control appearing linearly," in 2013 IEEE 52ND ANNUAL CONFERENCE ON DECISION AND CONTROL (CDC), ser. IEEE Conference on Decision and Control, 2013, pp. 514-519.

[15] L. Poggiolini and G. Stefani, "Structural stability for bang-singularbang extremals in the minimum time problem," SIAM J. Control Optim., vol. 51, no. 5, pp. 3511-3531, 2013. [Online]. Available: http://dx.doi.org/10.1137/120895421 